\title{
Calibration of Online Situation Awareness Assessment Systems Using Virtual Reality
}

\author{
Sebastien Mamessier, Daniel Dreyer, and Matthias Oberhauser \\ Airbus Group Innovations, Ottobrunn, Germany, \\ Creative Concept and Design Center, \\ Ottobrunn, Germany
}

\begin{abstract}
In an attempt to predict and prevent accident situations in complex socio-technical systems, one needs to be able to model and simulate concepts such as situation awareness (SA) and processes responsible for maintaining it. This is particularly true in the case of online support systems and adaptive displays which cannot rely on SA measurement techniques based on freeze probe techniques. This work investigates the state of the art in computational models of situation awareness and proposes a method to calibrate and evaluate such models using virtual reality human-in-the-loop experiments. This work introduces a new methodology to evaluate and calibrate online SA assessment systems taking advantage of the flexibility and reconfigurable power of virtual reality environments. This technology provides the experimenter with full control on the scenarios, cockpit types and interfaces. It also allows testing of off-nominal situations such as the loss of an instrument and more severe failures. Moreover, eye tracking capabilities provide an accurate way of registering monitoring events and feed SA assessment models with realistic data.
\end{abstract}

Keywords: situation awareness, mental models, virtual reality.

\section{Introduction}

In complex socio-technical environments such as modern aircraft cockpits, accidents still occur because of poorly designed human-integrated systems. Situation awareness of the pilot is often degraded by abnormally high workload or pilots' faulty mental models of the autoflight systems. Operators have to maintain an accurate representation of the situation supported by a good knowledge of the system's dynamics. Therefore, dangerous situations often occur when the complexity of the system or unexpected workload compromises the operator's situation awareness [31. In order to improve the automation's design or develop situation awareness-centered support systems [8], we need a methodology to evaluate the impact of design changes on SA as well as good indicators of $\mathrm{SA}$ for online assessment. The introduction of SA in the design loop assumes the development of SA assessment methods. However, SA is an abstract concept and its realization depends on many inputs such as the operator's mental 
model, the current workload and general monitoring and anticipating efforts. Methodologies such as SAGAT 9] provide a substantial help to measure SA and evaluate socio-technical systems. However most of these methods rely on "freezes" during simulations which makes them irrelevant to the design of online support systems. One needs computational models for the online assessment of SA based on live measurements to allow the existence of intelligent systems aiming to maintain and restore the operator's SA in real-time circumstances. This work introduces a methodology to evaluate and calibrate online SA assessment systems using virtual reality (VR) environments. VR cockpits can be modified easily and instruments hidden by the experimenter. Moreover, VR enables quick reconfiguration of the cockpit elements and can therefore be used to evaluate adaptive displays aimed to maintain or restore SA of the pilot.

\section{Background}

\subsection{Situation Awareness}

In 1988, Sarter and Woods introduced the concept of situation awareness without the support of an accurate definition [31. In 1995, Endsley [7] gave a formal definition and broke down SA into three different levels : perception (L1), comprehension (L2) and anticipation (L3). Endsley's theory is widely accepted across different fields of application such as aviation and nuclear safety as a critical concept to understand accidents and dangerous situations. Dekker et al. discussed the usefulness of what they call a folk model [3] : a consensual abstraction that hides the real phenomena. Whether SA is more an abstract product or a fundamental process is still discussed among human factor experts. Nevertheless, SA remains of great importance for safety [32] and methods for measuring SA have led to operational results.

Methodologies for measuring SA includes the Situation Awareness Global Assessment Technique (SAGAT) developed by Endsley [6] in which situation awareness is believed to be measurable by freezing a task [6] and simply querying the operator's understanding of the situation. SAGAT is a well validated method and is widely used to evaluate interfaces, and validate design changes such as new displays. Therefore it is often used in the design phase of the product lifecycle. However its intrusive nature prevents SAGAT from supporting the assessment of SA in real time circumstances. Other potential measurement techniques are described by Endsley in [8] involve psychophysiological metrics such as eye tracking data. They are described as appealing since unobtrusive and continuously available - thus suitable for live SA assessment - but are indirect and require a huge amount of calibration. Moreover the link between processes believed to maintain SA and SA remains unclear. Rare examples show that eye tracking can nevertheless give precious hints about the operator's situation awareness 28.33. Ratwani et al. trained a statistical model of SA capable of significantly predicting operators' ability to detect conflicting situations involving UAVs from eye-tracking data 28 . 


\subsection{SA-Based adaptive interfaces}

Safety critical concepts like SA should not only benefit to the design phase of complex systems. Indeed, testing and validation is limited to a finite set of scenarios and struggle at handling unexpected situations. Approaches such as Work Domain Analysis and Ecological Interface Design [35] strive for eventindependent design and are therefore helpful to extend the effectiveness of a design to off-nominal situations. Nevertheless, static interfaces are inherently limited since they don't capture the specific features of a situation.

Adaptive interfaces can change the position and saliency of different instruments during the operation. Changes can be driven by users' preferences, experience, fatigue, current task demands [30] or any other factors measurable by the system. Dehais et al. developed adaptive displays deploying cognitive countermeasures to prevent perseveration syndrome 2] and used performance-based validation metrics. Letsu and Ntuen 21 designed an adaptive interface for the DURESS II system [34].

Generally speaking, adaptive displays are the front-end of support systems that we believe should help maintain and restore situation awareness. Therefore there is a crucial need for online situation awareness computational models.

\subsection{Computational Models of SA Lack Validation}

Several computational models of situation awareness were developed around human models. Hoeey et al. modeled and predicted situation awareness of a human performance model of a pilot in [12. Their model included models of visual attention, perception and working memory but is limited to SA of discrete Situational Elements. Moreover, Hoeey et al. don't consider the impact of mental models, known to be one of the main factors responsible for maintaing SA [8]. Mc Carley et al. created an heuristic model for general SA accounting for attention and workload [27. This model was also used to predict the impact of dynamic SA display supports. However, SA is modeled as a unidimensional quantity which limits the adaptability power of the dynamic support interface. SAMPLE [36] is a fully integrated situation awareness prediction system of pilots involved in air combat. SAMPLE uses an extensive human model accounting for information processing and decision making. A Kalman filter is used to predict the pilot's estimate of continuous state variables and the pilot's knowledge of scenarios is represented as a bayesian network. Hanson et al. proposed to use SAMPLE as an online intelligent agent evaluating the pilot's situation assessment 11. However, SAMPLE does not benefit from online measurements such as eye tracking data but is only based on assumed monitoring patterns. In the field of interface design for nuclear plant operators, Lee and Seong [20] proposed a comprehensive model of SA including the effects of working memory decay, discrete mental models and instrument saliency. Mamessier et al. enhanced a computational human performance model [10] with situation awareness and mental model capabilities 25 23] including mental models of continuous and discrete dynamics. The model used in 2524 generates a multivariate belief function representing the current situation awareness of the operator. 
However few of these approaches use live measurements of the pilot's behavior to provide the model with realistic and online data. Furthermore many of these methods serve as an evaluation system for complex human-integrated designs but were not validated as such.

\section{Evaluation and Calibration of Online SA Assessment Systems}

Non-intrusive SA assessment systems with real-time constraints inherently rely on indirect measures of SA and therefore on an underlying model integrating them into a quantitative indicator of situation awareness. Therefore, such computational models of SA should be evaluated and calibrated.

\subsection{Requirements}

There are several factors that should be covered by any online SA assessment system. Jones et al. 14] analyzed 262 errors in 143 flight incidents. Around $50 \%$ of errors were caused by failures to observe data or detect important clues. $15 \%$ were related to incorrect mental models, the rest being distributed between unavailable data (13\%), forgotten data (8.5\%), misinterpretation $(8.7 \%)$ and other causes. Therefore computational models aiming to predict losses of SA and develop countermeasures should try to model and capture the operator's attention, data collection and account for mental models. Otherwise such models might overlook substantial evidence of loss of situation awareness.

\subsection{Process Indices}

Since non-intrusive SA assessment systems with real-time contraints cannot afford to query the pilot about his immediate knowledge, they rely on indirect measures. Psychophysiological measurement such as eye tracking methods are often referred to as Process indices as they don't directly measure SA but some of the processes contributing to the development of SA. Depending on the models and technology available, useful process indices could include recording of actual pilot actions, eye-tracking data, workload-related physiological measure, verbal communication between pilots. However, demonstrating which process indices are better for online SA assessment is not the main focus of this work. 29] emphasizes on their disadvantages, indirect nature and practical issues. The conclusion made in Salmon et al.'s extensive review of SA measurement methods 29] confirms SAGAT as as the most accurate and objective approach and overlooks methods based on process indices. The main idea behind this work is that we can evaluate online SA assessment systems by comparing their outputs with SAGAT objective measurements in a experimental setup. Instead of comparing Process indices and SAGAT as it was done in [298, we aim to use SAGAT as a widely recognized and validated SA measurement method to evaluate and calibrate indirect and model-based online SA assessments systems. 


\subsection{SA and Virtual Reality}

As listed above in the requirements, a SA assessment system should account for diverse processes such as attention/perception, mental models, workload and working memory decay. In order to evaluate and calibrate such complex models, one needs a flexible and reconfigurable environment providing the capability of decoupling the impact of influential factors. For instance, hiding instruments temporarily disconnects the perception loop to focus on the impact of mental models, normalizing the instruments' saliency enables the experimenter to limit the influence of design-dependent factors. Physical simulators are very constraining, onerous and too static to satisfy the needs of such an experiment.

On the other hand, virtual reality environments have many advantages. The assessment of SA within virtual environments was already theoretically discussed in the late nineties [5. Consequently, the original ideas of assessing SA in VR were picked up again by Matthews et al. [26] for the analysis of night vision goggles during dismounted soldier simulations. After each simulated night mission, the soldiers completed the Mission Awareness Rating Scale (MARS), an instrument designed to assess the subjective SA and workload experienced during a given mission. Laptaned 19 used tools like VRSAGAT, VRSARM and VRSART [15]16/17] in order to set SA, immersion, presence and performance in relation to each other. His research indicates a correlations between immersion and presence, and presence with SA. Horsch et al. [13] used Virtual Environments to test Urban Search and Rescue Robots. Their experiments showed no significant differences in SA and performance during several elementary tasks (e.g. slalom) between a virtual world and an experiment in reality. Recently, Dreyer et al. [4] conducted experiments in a Virtual Reality Flight Simulation and used NASA-TLX and SART questionnaires in order to assess workload and SA in different cockpit layouts.

\section{Virtual Reality Experiment}

A VR engine can load 3D models of aircraft cockpit as well as of a nuclear plant control room. The experiment presented here uses a VR aircraft cockpit and simulates flight scenarios involving a pilot. Since modern cockpits are considered as highly-sophisticated and complex human-integrated systems, we believe that the approach conveyed in this work can fit other domains as well as far as SA assessment systems are concerned.

\subsection{The Virtual Reality Flight Simulator}

The virtual reality environment used in this work is similar to the virtual reality flight simulator (VRFS) presented by Dreyer et al. 4]. This system is based on a consumer flight simulator that is extended in order to get VR capabilities. The actual position and orientation of the user's head is gathered via a head-tracking system and the viewpoint in the flight simulation software is adjusted accordingly [1]. With a three-dimensional cockpit geometry and a realistic outside view, 
both provided by the flight simulator, and a state of the art head mounted display (HMD) a highly immersive experience is provided. In addition, stereoscopic vision is achieved by slightly changing the camera positions for the right eye and the left eye. The use of hardware elements in the virtual reality flight simulator - e.g a throttle quadrant and a flight stick - creates a so called mixed mockup. To monitor the pilots' gaze behavior, an eye tracking system is part of the experimental setup. Commonly eye-tracking systems consist of an eye and a field camera. As the pilots eye is obscured the eye camera has to be mounted inside the HMD. To cover individual anatomic variations the camera position can be moved along a circular rail to fit most test persons [22]. Instead of a physical field camera, the visual content provided by the flight simulator must be used and is provided by a second synchronized instance of the flight simulator. Figure 1 shows the architecture of the virtual flight simulator adapted for the current evaluation.

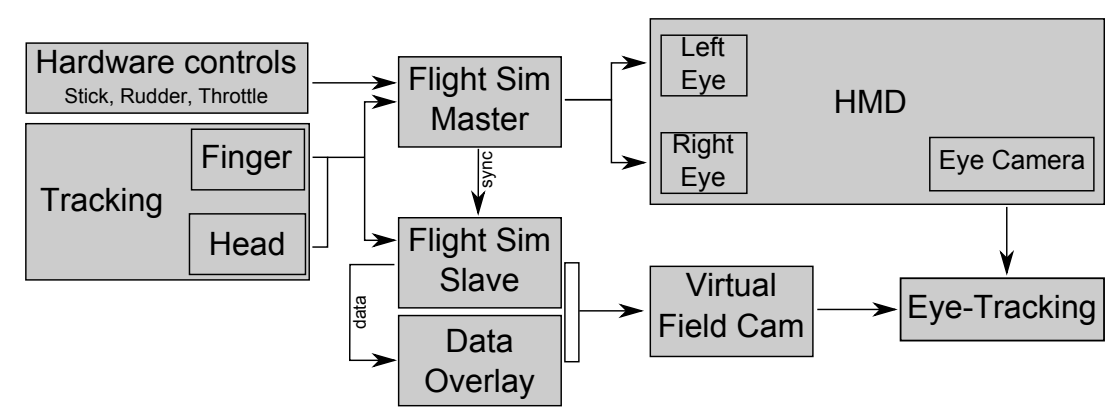

Fig. 1. Architecture of the Virtual Reality Flight Simulator

\subsection{Methodology}

As mentioned in 3.2 this work aims to use the well validated SAGAT method to assess the validity of online SA Assessment models and calibrate them. Depending on the assessment system, additional psychophysiological sensing capabilities may be required. We used eye-tracking information and live knowledge of the operator's actions as an online data stream to feed the model being evaluated. An example of both pilot view and experimenter view enhanced with eye-tracking sensing in shown in Figure 2. In parallel, the model makes an online assessment/prediction of the operator's situation awareness based on live data that we compare against direct and objective measurement using SAGAT-like questionnaires. To this end, the simulation is frozen at random times and instruments are hidden from the subject. The pilot is asked to describe his current knowledge of the situation, including important state variables and his/her degree of confidence. Fine calibration of the model with respect to mental models for example can be made by hiding instruments during a short period of time without freezing the simulation. 
The exact scenario and methodology depends on the model being evaluated. We believe that VR provides the experimenter with an increased flexibility enabling easier decoupling of influential variables. The analysis will be generally carried out by comparing the predictions issued by the online SA assessment system fed with live data and the SAGAT results.

\subsection{Example of Calibration}

Online SA Assessment Model. As a proof of concept, we chose to roughly calibrate the model of SA proposed by Mamessier and Feigh 2324 and used in the simulation of human-automation interaction in cockpit operations. This model focuses on the importance of modeling pilots' mental models of continuous and discrete dynamics of the aircraft. Situation awareness of continuous state variables such as altitude or airspeed is modeled as the result of merging direct monitoring events and model-based mental estimation. Kalman filter theory is used to model the data fusion process, building on Kleinman optimal control model of human response [18. The update phase of the model-based mental estimator was slightly modified for better incorporation of work memory decay and workload. The details of the changes mades are out of the scope of this paper and will be part of a future publication. The output of the model is a time-continuous gaussian probability distribution for each monitored variable (airspeed,altitude) representing the current believed value of the variable (the mean) and the operator's confidence about it. The operator's confidence is related to the distribution's standard deviation through a credible interval. Furthermore, Mamessier et al. model requires an approximation of the operator's mental model. We used the very simple mental model of altitude kinematics provided in [23] to fit our scenario needs. Moreover, a workload indicator between 0 and 1 was heuristically assumed from the pilot's activity and plugged into the model. Three parameters have to be calibrated representing respectively the impact of workload on memory decay rate, on the Kalman gain, and the credible interval.

Scenario. A standard instrument landing system (ILS) approach with a $3^{\circ}$ glide slope (GS) angle is chosen for this scenario. The flight starts at an altitude of $10200 \mathrm{ft}$ with the GS and the Localizer (LOC) already caught and centered, meaning the aircraft is perfectly on track. The autopilot is in the approach mode, roll and pitch of the aircraft are controlled by the autopilot. Besides the monitoring of the autopilot's flightpath the pilot has to manually control the airspeed using the throttle lever. To simulate different levels of workload, new airspeed clearances are verbally communicated to the pilot every 60 s. The pilot uses a Head-Up-Display (HUD) similar to the one studied by Dreyer in [4] and represented in Figure 2. Freezing times are generated by a gaussian incremental process with inter-freeze times sampled from $\mathcal{N}\left(30 s, 100 s^{2}\right)$ to prevent the subject to anticipate the freezing schedule. 

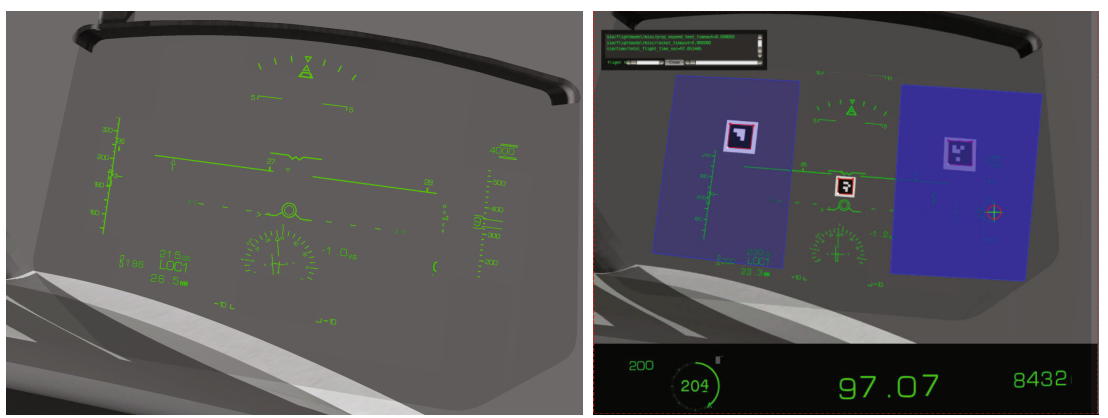

Fig. 2. Pilot and experimenter view

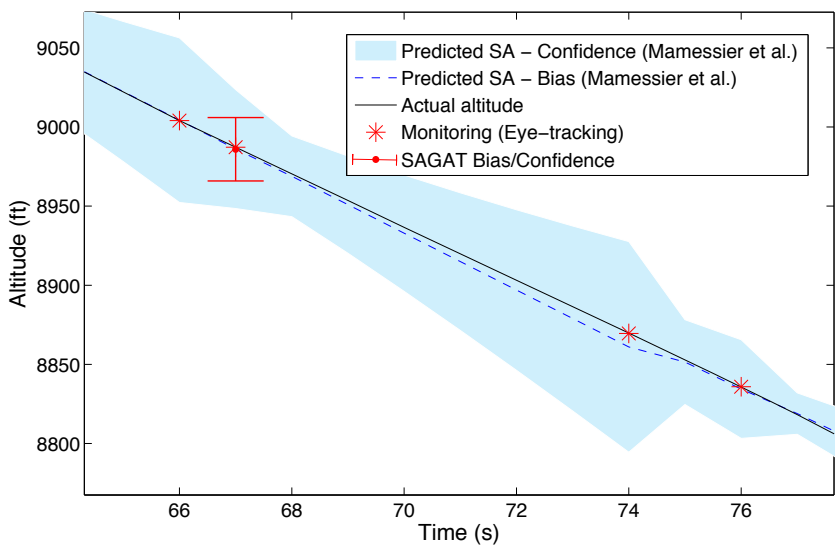

Fig. 3. Predicted Situation Awareness of the altitude (blue) based on the detection of online monitoring events (red stars) compared against SAGAT answers (red bars and dots)

Example of Calibration Data. Figure 3 shows an example of output of the SA online assessment system inspired from Mamessier et al., replacing simulated monitoring patterns with realistic monitoring events obtained with eye-tracking data. The black line is the actual altitude of the aircraft. The red stars represent the very times at which the pilot checked the altimeter and red bars stand for the SAGAT freezes results. The bar's length represents the pilot's confidence about his answer and the red dot his belief of the altitude. The dotted blue line is the predicted pilot's altitude belief and the light blue area the predicted credible interval.

The C-credibility interval of the gaussian bayesian belief of variable $x_{i}$ is $I_{C \%}=\left[x^{i} \pm \sqrt{2} \sigma^{i} \operatorname{erf}^{-1}\left(\frac{C}{100}\right)\right][25]$. It means that the operator is $\mathrm{C} \%$ sure that the actual value is within $I_{C}$. In order to compare the subject's SAGAT results about confidence and credible intervals predicted by the model, we need to pick a value for $\mathrm{C}$. This value can be calibrated by aligning the smallest $I_{C}$ predicted 


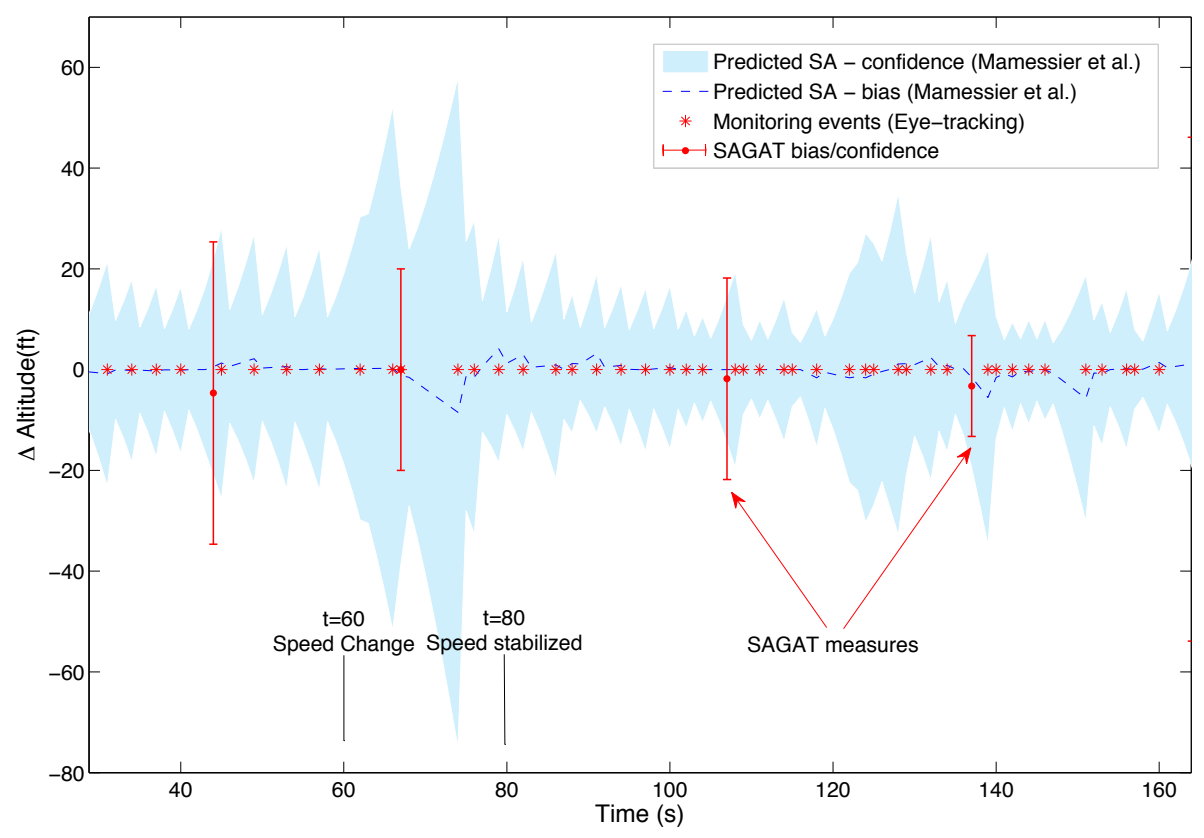

Fig. 4. Pilot's estimation bias of the altitude, recentered on the actual altitude $(\Delta$ Altitude $=0)$ and credible intervals obtained by feeding Mamessier et al.'s model with online eye-tracking data (monitoring events) and compared against SAGAT queries

by the model along the flight and the subject's best confidence across SAGAT freezes. In this example, we found that $C=85 \%$ led to a good calibration.

Figure 4 represents both online predicted and SAGAT-measured situation awareness of the pilot recentered on the actual aircraft altitude. Workload peaks and predicted consequences on the operator's SA can be observed between 60 and $80 \mathrm{~s}, 120 \mathrm{~s}$ and $140 \mathrm{~s}$ where the pilot had to change and stabilize the airspeed. The actual (SAGAT) and predicted pilot's bias (difference between believed altitude and actual altitude) is close to zero most of the time due to a high monitoring frequency. We observe a short increase in the absolute predicted bias around 70s due to the temporary absence of monitoring and the change of speed. Indeed, vertical speed slightly increased due to the speed change making the operator's mental model inaccurate until more monitoring finally allows the pilot to adapt.

\section{Conclusion}

Due to the increasing complexity of socio-technological systems, operators' situation awareness has become a critical concept for safety. Using SA measurement methods like SAGAT in the design loop of such systems is crucial to improve human-automation interaction. However non-intrusive online SA assessment is 
needed to cope with unpredictable situations through live support systems such as adaptive displays. To that end, we introduced a methodology based on the well validated SAGAT method and on the reconfigurable power of virtual reality to evaluate and calibrate online SA assessment systems and models based on SA process indices measurements. Mamessier et al. model was used as an example and was connected to a virtual reality flight simulator and eye-tracking system. Our experimental setup turned out to be very flexible and already provided many promising insights to extend and improve online SA assessment models. Based on this example, future work will include a full-scale statistical analysis and calibration of a SA online assessment model with professional pilots. Moreover, adaptive displays based on calibrated SA assessment models can be evaluated with the same experimental setup using the flexibility of virtual reality.

\section{References}

1. Aslandere, T.I.: Interaction Methods in a Generic Virtual Reality Flight Simulator. Master's thesis, Technische Universitat Munchen (2013)

2. Dehais, F., Tessier, C., Christophe, L., Reuzeau, F.: The perseveration syndrome in the pilots activity: Guidelines and cognitive countermeasures. In: Palanque, P., Vanderdonckt, J., Winckler, M. (eds.) HESSD 2009. LNCS, vol. 5962, pp. 68-80. Springer, Heidelberg (2010)

3. Dekker, S., Hollnagel, E.: Human factors and folk models. Cognition, Technology \& Work 6(2), 79-86 (2004)

4. Dreyer, D., Bandow, D., Oberhauser, M.: Hud symbology evaluation in a virtual reality flight simulation. Paper submitted to HCI Aero 2014, Silicon Valley, USA (2014)

5. Ehrlich, J., Knerr, B., Lampton, D., McDonald, D.: Team situational awareness training in virtual environments: Potential capabilities and research issues. Tech. rep., U.S. Army Research Institute for the Behavioral and Social Sciences (1997)

6. Endsley, M.R.: Measurement of situation awareness in dynamic systems. Human Factors: The Journal of the Human Factors and Ergonomics Society 37(1), 65-84 (1995)

7. Endsley, M.R.: Toward a theory of situation awareness in dynamic systems. Human Factors: The Journal of the Human Factors and Ergonomics Society 37(1), 32-64 (1995)

8. Endsley, M.R.: Designing for situation awareness: An approach to user-centered design. Taylor \& Francis, US (2003)

9. Endsley, M.: Situation awareness global assessment technique (sagat). In: Proceedings of the IEEE 1988 National Aerospace and Electronics Conference, NAECON 1988, pp. 789-795. IEEE (1988)

10. Feigh, K.M., Gelman, G., Mamessier, S., Pritchett, A.R.: Simulating first-principles models of situated human performance. IEEE Transactions on System Man and Cybernetics: Part A (submitted June 2012)

11. Hanson, M.L., Sullivan, O., Harper, K.A.: On-line situation assessment for unmanned air vehicles. In: FLAIRS Conference, pp. 44-48 (2001)

12. Hooey, B.L., Gore, B.F., Wickens, C.D., Scott-Nash, S., Socash, C., Salud, E., Foyle, D.C.: Modeling pilot situation awareness. In: Human Modelling in Assisted Transportation, pp. 207-213. Springer, Heidelberg (2011) 
13. Horsch, C., Smets, N., Neerincx, M., Cuijpers, R.: Comparing performance and situation awareness in usar unit tasks in a virtual and real environment. In: Proceedings of the 10th International ISCRAM Conference, Baden-Baden, Germany, pp. 556-560 (2013)

14. Jones, D.G., Endsley, M.R.: Sources of situation awareness errors in aviation. Aviation, Space, and Environmental Medicine (1996)

15. Kalawsky, R.: New methodologies and techniques for evaluating user performance in advanced 3d virtual interfaces. IEEE Colloquium Digest 98/43 (1998)

16. Kalawsky, R.: Vruse - a computerised diagnostic tool for usability evaluation of virtual/synthetic environment systems. Applied Ergonomics 30, 11-25 (1999)

17. Kalawsky, R., Bee, S., Nee, S.: Human factors evaluation techniques to aid understanding of virtual interfaces. BT Technology Journal 17(1), 128-141 (1999)

18. Kleinman, D., Baron, S., Levison, W.: An optimal control model of human response part i: Theory and validation. Automatica 6(3), 357-369 (1970)

19. Laptaned, U.: Situation awareness in virtual environments: A theoretical model and investigation with different interface designs. In: Proceedings of the 9th IASTED International Conference Computers and Advanced Technology in Education, Lima, Peru, pp. 277-283 (2006)

20. Lee, H.C., Seong, P.H.: A computational model for evaluating the effects of attention, memory, and mental models on situation assessment of nuclear power plant operators. Reliability Engineering \& System Safety 94(11), 1796-1805 (2009)

21. Letsu-Dake, E., Ntuen, C.A.: A case study of experimental evaluation of adaptive interfaces. International Journal of Industrial Ergonomics 40(1), 34-40 (2010)

22. Liesecke, S.: Eye-Tracking in Virtual Reality. Master's thesis, Universitt der Bundeswehr Munchen (2013)

23. Mamessier, S.: A computational approach to situation awareness and mental models in aviation. Master's thesis, Georgia Institute of Technology (2013)

24. Mamessier, S., Feigh, K.: Simulating the impact of mental models on human automation interaction in aviation. In: Duffy, V.G. (ed.) DHM/HCII 2013, Part I. LNCS, vol. 8025, pp. 61-69. Springer, Heidelberg (2013)

25. Mamessier, S., Feigh, K.: A computational approach to situation awareness and mental models for continuous dynamics in aviation. IEEE Transactions on HumanMachine Systems, (2014) (Under review)

26. Matthews, M., Beal, S., Pleban, R.: Situation awareness in a virtual environment: Description of a subjective assessment scale. Tech. rep. U.S. Army Research Institute for the Behavioral and Social Sciences (2002)

27. McCarley, J.S., Wickens, C.D., Goh, J., Horrey, W.J.: A computational model of attention/situation awareness. In: Proceedings of the Human Factors and Ergonomics Society Annual Meeting, vol. 46, pp. 1669-1673. SAGE Publications (2002)

28. Ratwani, R.M., McCurry, J.M., Trafton, J.G.: Single operator, multiple robots: An eye movement based theoretic model of operator situation awareness. In: 2010 5th ACM/IEEE International Conference on Human-Robot Interaction (HRI), pp. 235-242. IEEE (2010)

29. Salmon, P., Stanton, N., Walker, G., Green, D.: Situation awareness measurement: A review of applicability for c4i environments. Applied Ergonomics 37(2), 225-238 (2006)

30. Sarter, N.: Coping with complexity through adaptive interface design. In: Jacko, J.A. (ed.) Human-Computer Interaction, Part III, HCII 2007. LNCS, vol. 4552, pp. 493-498. Springer, Heidelberg (2007)

31. Sarter, N.B., Woods, D.D.: Situation awareness: A critical but ill-defined phenomenon. The International Journal of Aviation Psychology 1(1), 45-57 (1991) 
32. Stanton, N.A., Chambers, P., Piggott, J.: Situational awareness and safety. Safety Science 39(3), 189-204 (2001)

33. Tien, G., Atkins, M.S., Zheng, B., Swindells, C.: Measuring situation awareness of surgeons in laparoscopic training. In: Proceedings of the 2010 Symposium on Eye-Tracking Research \& Applications, pp. 149-152. ACM (2010)

34. Vicente, K., Pawlak, W.: Cognitive work analysis for the duress ii system. Cognitive Engineering Laboratory, Department of Industrial Engineering, Toronto, Canada CEL, pp. 94-93. University of Toronto (1994)

35. Vicente, K.: Cognitive work analysis: Toward safe, productive, and healthy computer-based work. Lawrence Erlbaum (1999)

36. Zacharias, G.L., Miao, A.X., Illgen, C., Yara, J.M., Siouris, G.M.: Sample: Situation awareness model for pilot-in-the-loop evaluation. Final Report R 95192 (1996) 